\title{
Encondroma protuberans de falange en niño. A propósito de un caso
}

\author{
A. López-Hualda ${ }^{(1)}$, H. Fahandezh-Díaz ${ }^{(2)}$, R. M. Egea-Gámez ${ }^{(1)}$, \\ F. Panizo-Mota ${ }^{(1)}$, J. E. Ruiz-Zafra ${ }^{(2)}$ \\ Hospital Universitario Fundación Alcorcón. Unidad de Mano y Miembro Superior \\ (1) Médico Interno Residente de Cirugía Ortopédica y TRaumatología \\ (2) Unidad de Cirugía de la MANO Y ExTREMIDAd SUPERIoR
}

\author{
Correspondencia: \\ Dr. Álvaro López Hualda \\ Hospital Universitario Fundación Alcorcón \\ Servicio de Cirugía Ortopédica y Traumatología \\ C/ Budapest, $\mathrm{n}^{\circ} 1$ \\ 28922 Alcorcón (Madrid) \\ Teléfono: 916219400 \\ e-mail: alhualda@ hotmail.com
}

\begin{abstract}
El encondroma protuberans es una entidad rara, solo se han descrito 14 casos en la bibliografía. Radiológicamente puede presentar dudas diagnósticas con un osteocondroma, condrosarcoma de bajo grado y condroma periostal. Presentamos el caso de un niño de cinco años con un encondroma protuberans en falange proximal que experimentó un crecimiento acelerado durante su seguimiento, además presentaba dos encondromas convencionales en la misma mano. Fue tratado mediante resección marginal, curetaje y relleno con autoinjerto óseo, sin evidencia de recidiva a los 12 meses de seguimiento.
\end{abstract}

Palabras clave: encondroma protuberans, falange, mano.
Enchondroma protuberans is a rare entity, there have been 14 previus cases reported. Morphologic features seen on radiographs could mistake us with other tumours like osteochondroma, low level chondrosarcoma and periosteal chondroma.

We present a case of a 5-year-old boy with an acelerated gowth of an enchondroma protuberans in proximal phalanx, associated with two conventional enchondroma in the same hand. The patient was treated with marginal excision, curettage and the placement of autograft, there was no evidence of recurrence at 12 month follow up examination.

Key words: enchondroma protuberans, phalanx, hand.

\section{INTRODUCCIÓN}

1 1 encondroma protuberans, es un tumor benigno, raro, de origen condral ${ }^{(1)}$. Difiere del encondroma convencional en que presenta una tendencia a expandirse exofíticamente desde la medular de los huesos largos, preferentemente en las extremidades superiores. Presentamos el caso de un encondroma protuberans que experimentó un crecimiento acelerado durante su seguimiento. Además discutiremos el diagnóstico y tratamiento de dicha lesión tras realizar una revisión sistemática de la bibliografía.

\section{CASO CLÍNICO}

Varón de 5 años que acude a la consulta de ortopedia del hospital presentando una tumoración no dolorosa en segundo dedo de la mano 


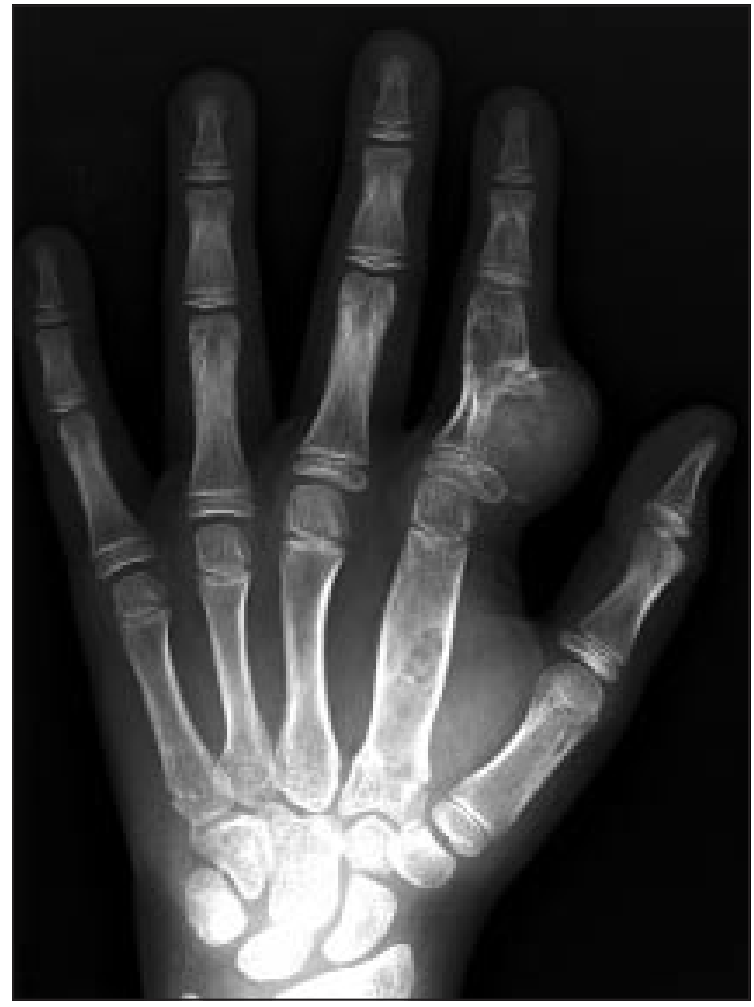

Figura 1: Radiología simple de mano izquierda. Rx preoperatoria. Se aprecia la lesión osteolítica que expande excéntricamente la cortical radial de la falange proximal del $2^{\circ}$ dedo.

izquierda. Cinco años después, se objetivó un crecimiento acelerado de dicha lesión alcanzando los $3 \mathrm{~cm}$ de diámetro. La lesión no ocasionaba síntomas y no limitaba la movilidad del dedo.

La radiología simple mostró una lesión osteolítica excéntrica con ruptura de la cortical radial de la falange proximal, con márgenes bien definidos. Además presentaba otras dos lesiones osteolíticas endomedulares con cortical conservada a nivel de la falange media del mismo dedo y en el segundo metacarpiano de la misma mano (Figura 1).

Se realizó una gammagrafía con tecnecio 99, que evidenció captación de las lesiones de las falanges y del metacarpiano, descartándose la existencia de otros focos a distancia. Finalmente se completó el estudio con una resonancia magnética ( $\mathrm{RM})$, en la que la lesión de la falange proximal se presentaba hipointensa en $\mathrm{T} 1$ con un tamaño de 25x19x16 mm, producía un desplazamiento de tendones flexores y paquete vasculonervioso, sin invasión de dichas estructuras (Figura 2).

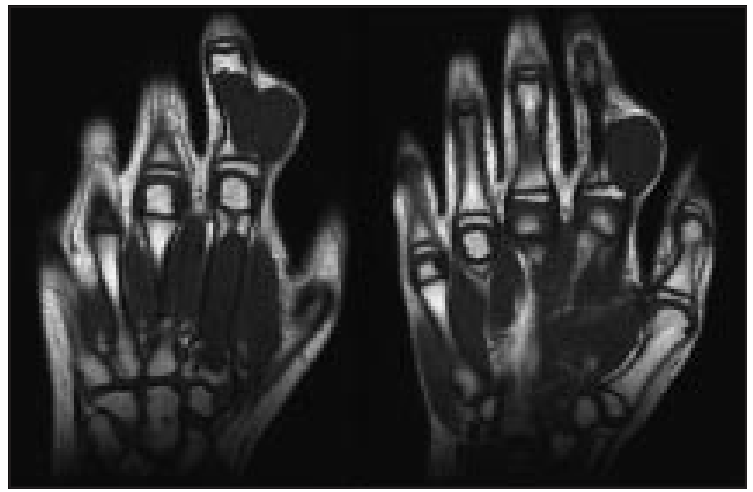

Figura 2: Resonancia magnética de mano izquierda. Corte coronal en secuencia T1. Encondroma protuberans en falange proximal y encondroma convencional en segundo metacarpiano de la misma mano.

El paciente fue tratado mediante resección de la lesión prominente y curetaje del lecho (Figura 3). El defecto óseo fue rellenado con injerto óseo autólogo de cresta iliaca.

Desde el punto de vista anatomopatológico, la lesión estaba constituida por cartílago hialino con un patrón lobular, presentaba condrocitos con núcleos aumentados de tamaño, binucleación y cambios mixoides del estroma. Lesión era compatible con un encondroma (Figura 4).

A los 12 meses de la intervención, el paciente se encontraba asintomático, sin evidencia clínica ni radiológica de recidiva de la lesión, ni progresión de las lesiones adyacentes (Figura 5). Presentaba una movilidad completa con buena funcionalidad.

\section{DISCUSIÓN}

El encondroma protuberans es una entidad rara, tan sólo se han publicado catorce casos en la bibliografía ${ }^{1}$. La localización más frecuente es en falanges y metacarpianos (8 casos), aunque también se han descrito 3 casos en húmero y 3 en costillas ${ }^{2}$. La edad de aparición suele estar comprendida entra la $2^{\mathrm{a}}-5^{\mathrm{a}}$ década de la vida.

Radiológicamente aparece como una lesión osteolítica de márgenes bien definidos, que se expande excéntricamente en la región metafisodiafisaria de huesos largos con frecuente ruptura de la cortical. En el espesor de la lesión suelen ser frecuentes calcificaciones puntiformes. En la RM aparece característicamente como una 


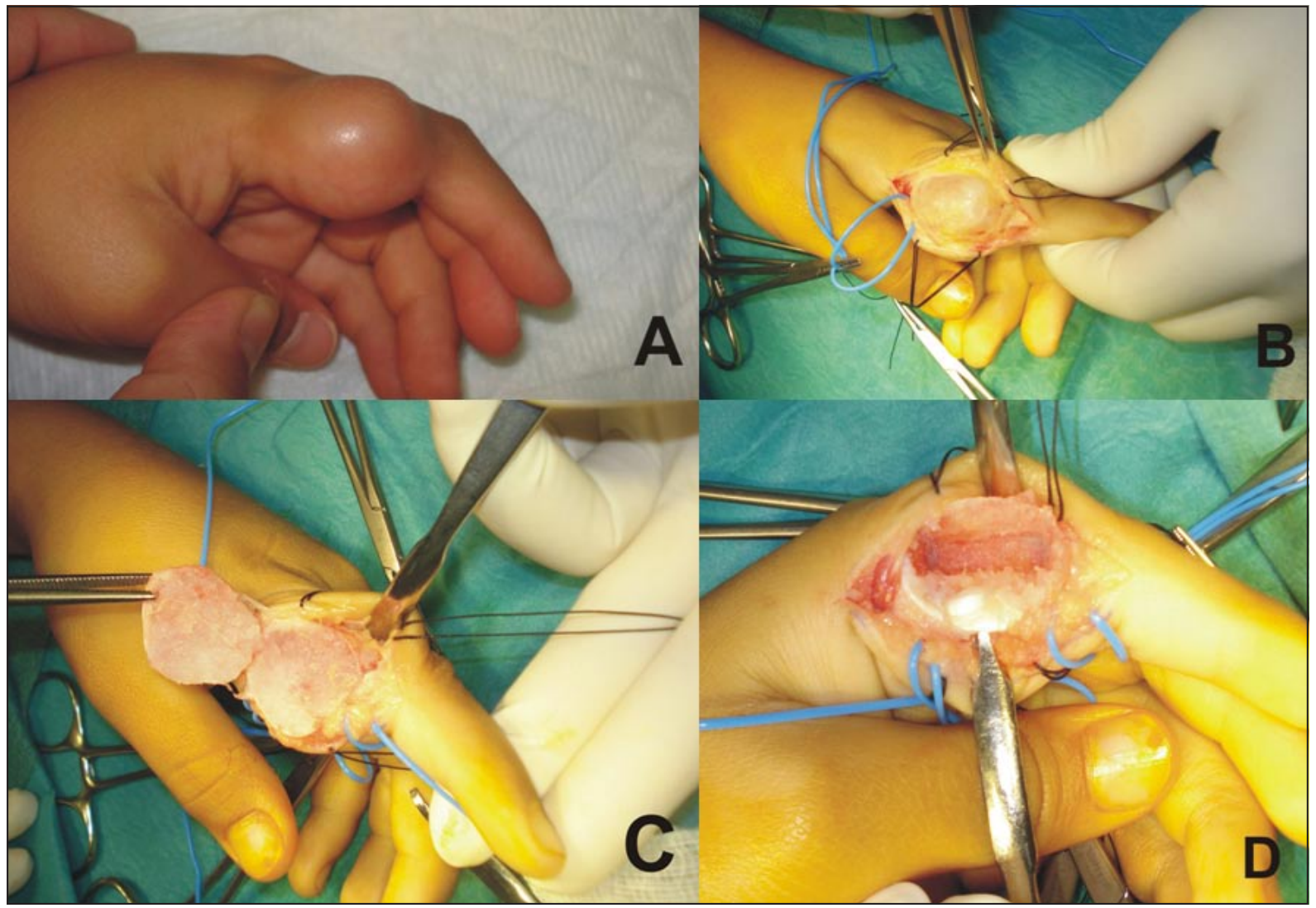

Figura 3: Secuencia quirúrgica. (A) Imagen preoperatoria. (B) Identificación de paquete vásculo-nervioso respetado por la lesión. (C) Biopsia excisional de la lesión. (D) Curetaje de la lesión.

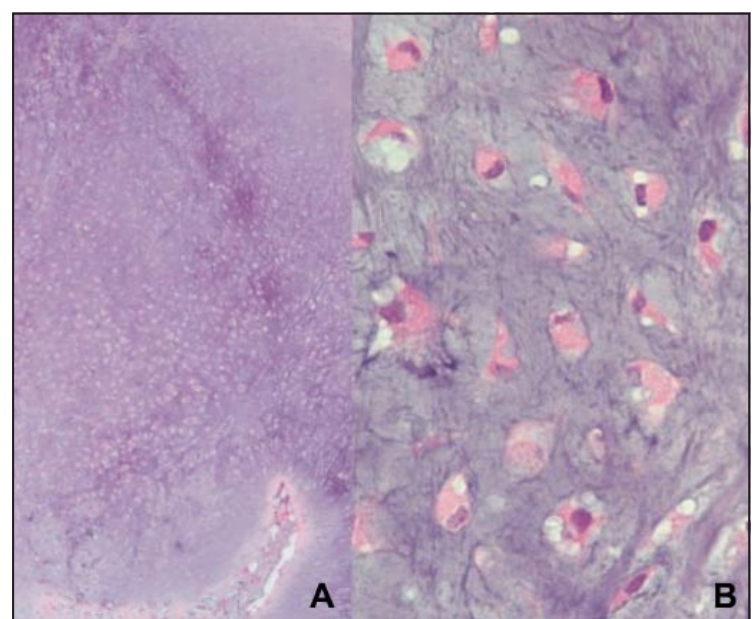

Figura 4: Visión microscópica de encondroma protuberans con hematoxilina-eosina $x 40$ (A) y $x 400$ (B). La lesión está constituida por cartílago hialino con un patrón lobular. Se aprecia hipercelularidad, condrocitos con núcleos aumentados de tamaño, presencia de binucleación y cambios mixoides del estroma.

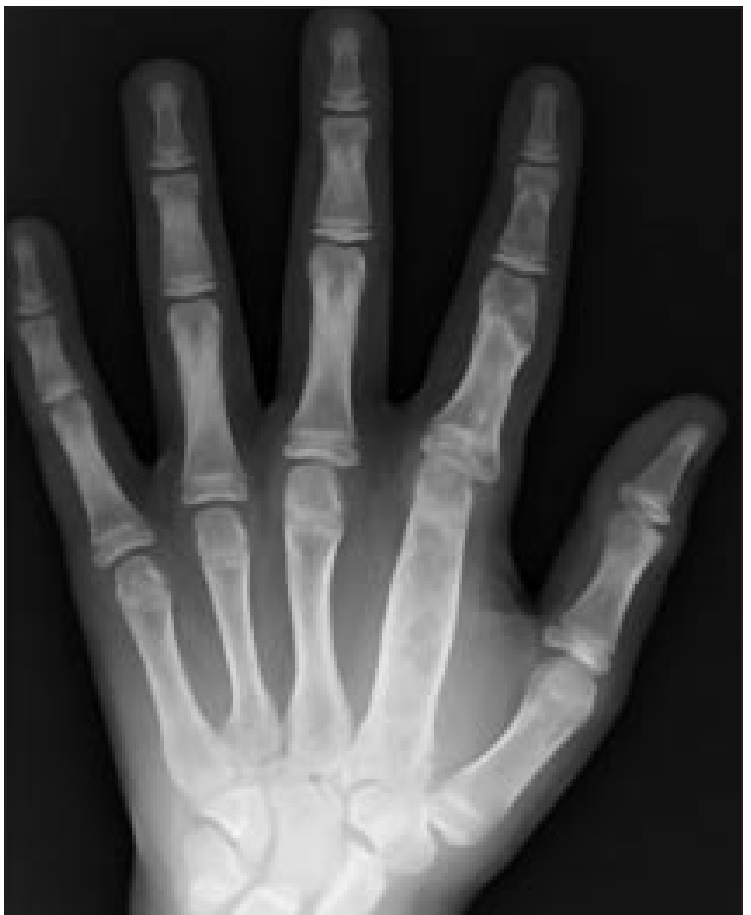

Figura 5: Un año después de la cirugía no se evidencia signos de radiológicos de recidiva. 
lesión lobulada expansiva hipointensa en T1 de forma constante, sin invasión de estructuras adyacentes ${ }^{3}$.

El diagnóstico diferencial debe plantearse con otros tumores de la mano con patrón osteolítico como son osteocondroma, condrosarcoma de bajo grado y condroma periostal ${ }^{1,3}$. Una evolución agresiva con crecimiento rápido y dolor asociado, ruptura de corticales, destrucción de la matriz condral por aumento del componente no mineralizado e invasión de los tejidos blandos adyancentes son datos que apoyan una malignización de la lesión o un diagnóstico distinto del encondroma protuberans. Así la evolución clínica y la RM nos darán la clave a la hora de realizar el diagnóstico.

El tratamiento consiste en curetaje de la lesión y relleno del defecto óseo con cemento de fosfato cálcico o injerto óseo ${ }^{1-4}$. Aunque no existe ningún estudio que establezca diferencias entre ambas opciones, la mayoría autores optan por el autoinjerto. Es importante realizar un adecuado curetaje del lecho, dado que si éste fuese deficitario puede favorecer la recidiva ${ }^{3}$. En nuestro caso se optó por utilizar injerto óseo autólogo consiguiendo buenos resultados en la integración, sin evidenciarse signos de recidiva al año de evolución.

\section{BIBLIOGRAFÍA}

1. An YY, Kim JY, Ahn MI, Kang YK, Choi HJ. Enchondroma protuberans of the hand. AJR. Am J Roentgenol, 2008; 190: 40-4.

2. Degreef I, De Smet L. Enchondroma protuberans of the phalanx. Scand J Plast Reconstr
Surg Hand Surg, 2005; 39: 315-7.

3. Slesarenko YA, Sampson SP, Gould ES, Dagum AB. Recurrent enchondroma protuberans: a case report. J Hand Surg Am, 2005; 30: 1318-21.
4. Kita K, Masada K, Yasuda M, Takeuchi E. Enchondroma protuberans of the phalanx: a case report. J Hand Surg Am, 2003; 28: 1052-4. 